\title{
Individual Income Status Correlates with Chronic Kidney Disease in Japan beyond Metabolic Risk Factors: Cross Sectional Study
}

\author{
Hoichi Amano ${ }^{1,2 *}$, Yoshiharu Fukuda1, Chiho Kitashima1, Takashi Yokoo², Kazue Yamaoka1 \\ ${ }^{1}$ Graduate School of Public Health, Teikyo University, Tokyo, Japan \\ ${ }^{2}$ Division of Nephrology and Hypertension, Department of Internal Medicine, The Jikei University School of Medicine, Tokyo, \\ Japan \\ Email: ^m04002.belongto@gmail.com
}

How to cite this paper: Amano, H., Fukuda, Y., Kitashima1, C., Yokoo, T. and Yamaoka, K. (2017) Individual Income Status Correlates with Chronic Kidney Disease in Japan beyond Metabolic Risk Factors: Cross Sectional Study. Health, 9, 1516-1528.

https://doi.org/10.4236/health.2017.911112

Received: September 27, 2017

Accepted: October 24, 2017

Published: October 27, 2017

Copyright ( 2017 by authors and Scientific Research Publishing Inc. This work is licensed under the Creative Commons Attribution International License (CC BY 4.0).

http://creativecommons.org/licenses/by/4.0/

\begin{abstract}
Background: Chronic kidney disease (CKD) is a pre-condition for end-stage kidney disease (ESKD) undergoing hemodialysis, as well as a risk factor for cardiovascular disease. Therefore, it is vital that CKD prevention measures be established. In particular, socioeconomic status (SES), as represented by income, contributes to non-communicable diseases like CKD. The purpose of this study was to examine the independent association between CKD and individual SES in Japan, with consideration of other metabolic risks. Methods: In the present study, we analyzed the 2011 National Health and Nutrition Survey by the Ministry of Health, Labor, and Welfare. Specifically, we analyzed 3,557 people out of 8,762 whose serum creatinine was measured. Logistic regression analysis was used to calculate the odds ratio (OR) of CKD by income distinction, after adjustment for age and metabolic risks (obesity, diabetes mellitus, dyslipidemia, and hypertension), which were assessed at the medical examination. Results: CKD was found in 385 participants (10.8\%) and was associated with greater age, obesity, higher levels of low-density lipoprotein cholesterol, triglyceride, and glycated hemoglobin, and lower levels of hemoglobin and high-density lipoprotein cholesterol. The adjusted model indicated a significant association between lower income and CKD: the OR of the low income group ( $<2$ million yen) was 1.33 (95\% confidence interval: 1.01 - 1.78) in comparison with the high income group. Conclusions: The results of the present study indicate a substantial relationship between individual lower income status and CKD in Japan, where healthcare is easy to access. To prevent ESKD and cardiovascular diseases, early detection of CKD and its metabolic risks is necessary, especially among the socioeconomically vulnerable population.
\end{abstract}




\section{Keywords}

Chronic Kidney Disease, End-Stage Kidney Disease, Socioeconomic Status, Income, Population Approach

\section{Introduction}

The number of patients with end-stage kidney disease (ESKD) undergoing hemodialysis has been increasing in most parts of the world, and the worldwide medical expenses associated with hemodialysis will increase to 1.1 billion US dollars within the next 10 years [1]. In Japan, the number of patients undergoing hemodialysis has tripled in the past 20 years, and dialysis-related medical expenses now amount to 1.4 billion yen [2] [3].

In 2002, a new disease concept, chronic kidney disease (CKD), was proposed. In $\mathrm{CKD}$, kidney function disorder and/or abnormalities in urine appearance continue for more than 3 months, regardless of cause. Early detection and treatment of CKD may prevent progression to ESKD. In fact, CKD is a pre-condition for ESKD, as well as a risk factor for cardiovascular disease (CVD), which is the main cause of death worldwide [4] [5] [6]. It is estimated that $13 \%$ of the Japanese population have CKD; thus, the disease is as common as diabetes mellitus (DM) and hypertension (HT). For this reason, it is vital that effective CKD preventive measures be established; in fact, this is one of the most important issues in public and national health.

Several previous studies have evaluated the metabolic and lifestyle risk factors of CKD [2] [7] [8] [9] [10]. Metabolic factors, including DM, dyslipidemia (DL), $\mathrm{HT}$, and obesity are major risk factors for CKD. In addition, physical inactivity and insufficient sleep time have been demonstrated as risk factors [10] [11]. Several studies have suggested that excess alcohol consumption is also a risk factor, although results are inconsistent in this regard [12]. Taken together, the accumulation of unhealthy lifestyle factors strongly increases the risk of CKD [13].

Medical examination and easy access to medical care play an important role in the early detection and prevention of CKD. In the guidelines "Proposal to achieve a decline in the number of dialysis initiations due to lifestyle-related diseases-early detection and prevention of onset and progression of CKD", which was published by the Japanese Society of Nephrology in 2016, the importance of regular medical examinations for the early detection of CKD is emphasized. In Japan, specific health check-ups and health-maintenance controls are carried out nationwide to prevent non-communicable diseases, especially metabolic syndrome. These programs aim to restrict increases in medical expenses; however, they have not yet focused on preventing CKD.

Recently, researchers have focused on whether socioeconomic status (SES) is associated with metabolic risk factors, unhealthy lifestyle, and CKD. The term 
"SES" refers to the differences between individuals and groups in the possession of resources. The major indicators of SES are individual/household income, education, and occupation. Furthermore, SES reportedly contributes to both unhealthy lifestyle and CKD [14] [15], and it is a risk factor for mortality in patients with CKD [16]. Despite this, to our knowledge, the association between CKD and SES in Japan is still not completely understood.

The purpose of the present study was to examine the association between $\mathrm{CKD}$ and individual income status in Japan using a nation representative sample, with consideration for other metabolic risk factors. The hypothesis of the present study is shown in Figure 1. SES is associated with CKD, mainly as a mediator of metabolic and lifestyle risks. The indicator of SES used in the present study was individual income, and lifestyle factors were regular alcohol intake, physical inactivity and insufficient sleep time.

\section{Subjects and Methods}

\subsection{Data Source}

In the present study, we analyzed the 2011 National Health and Nutrition Survey by the Ministry of Health, Labor, and Welfare, which was a random sampling survey representing Japanese population distribution. Based on the Health Promotion Act, the National Health and Nutrition Survey is conducted annually by the Ministry of Health, Labor, and Welfare to evaluate people's health, nutritional intake, and lifestyle habits, and to obtain the basic data necessary for comprehensive health promotion. All members of households within 300 area units were interviewed. These units were randomly selected from among approximately 11,000 area units, as defined in the Comprehensive Survey of Living Conditions, 2011. More specifically, the survey recorded individual demographic information, illness profile, and lifestyle. In addition, a health examination was

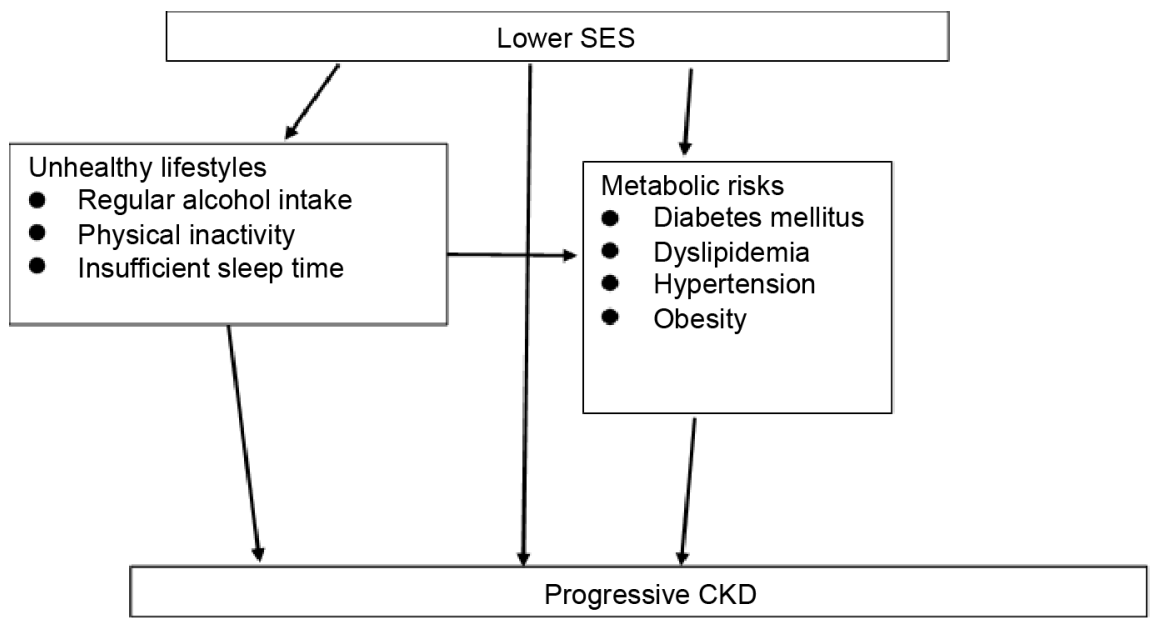

Figure 1. There is the association between lower socioeconomic statues (SES) and unhealthy lifestyles or metabolic risks. Furthermore, unhealthy lifestyles, metabolic risks and uncollected factors might be associated with chronic kidney disease (CKD) progression. But in Japan, the association between CKD and SES is still not completely understood. 
carried out that included anthropometry, blood pressure measurement, and a blood test. The details of the survey have been reported elsewhere [17]. We obtained permission to use these data from the Ministry of Health, Labor, and Welfare.

\subsection{Clinical Data and Disease Definition}

The following four disease definitions were used in the analysis: CKD, DM, DL, and HT. CKD was defined as an estimated glomerular filtration rate (eGFR) $<60$ $\mathrm{ml} / \mathrm{min} / 1.73 \mathrm{~m}^{2}$. The eGFR was estimated using the following formula: eGFR $\left(\mathrm{ml} / \mathrm{min} / 1.73 \mathrm{~m}^{2}\right)=(194 \times \mathrm{Cr})-(1.094 \times$ age- 0.287$)(\times 0.739$, if female $)$. This formula was proposed by the Japanese Society of Nephrology [2]. DM was defined as either (1) HbA1c of $\geq 6.5 \%$, as described by the National Glycohemoglobin Standardization Program, and/or (2) use of antidiabetic medications [18]. DL was defined as either (1) low density lipoprotein cholesterol (LDL-C) $\geq 140$ $\mathrm{mg} / \mathrm{dl}$, (2) high density lipoprotein cholesterol (HDL-C) $<40 \mathrm{mg} / \mathrm{dl}$, (3) triglyceride (TG) $\geq 150 \mathrm{mg} / \mathrm{dL}$, and/or (4) the use of cholesterol-lowering drugs [19]. HT was defined as either (1) systolic blood pressure (SBP) $\geq 140 \mathrm{mmHg}$, (2) diastolic blood pressure (DBP) $\geq 80 \mathrm{mmHg}$, and/or (3) use of antihypertensive drug [20]. Body mass index (BMI) was calculated using the subjects' height and weight, and obesity was defined as a $\mathrm{BMI} \geq 25$.

\subsection{Health Risk Behavior}

The following three behavioral health risks were used in the analysis: regular alcohol intake, physical inactivity and insufficient sleep time. Alcohol consumption per week was surveyed using the question, "How many times do you drink per week?" Regular alcohol intake was defined as drinking once or more per week. Physical inactivity was defined when the participants had "no exercise custom" in the original questionnaire. Insufficient sleep time was defined as 5 or less hours of sleep per day.

\subsection{Individual Income}

We used income as an indicator of individual SES, because other indications of SES, such as education and occupation, could not be collected. Participants were asked to select their income from a list of three categories: $<2$ million yen, 2 million to $5,999,999$ yen, or $\geq 6$ million yen. For the analysis, two categories of income were used: $<2$ million yen and $\geq 2$ million yen.

\subsection{Statistical Analysis}

We analyzed 3,557 out of 8,762 people whose serum creatinine ( $\mathrm{Cr}$ ) had been measured. Differences in independent variables between CKD and non-CKD were assessed using the Chi-square test in the case of categorical variables and the t-test in the case of continuous variables in Table 1. Analysis about differ-

ences between income was done in the same way. In several studies addressing 
Table 1. Basic characteristics of the participants in terms of the presence of CKD.

\begin{tabular}{|c|c|c|c|}
\hline Variables & Non-CKD $(\mathrm{n}=3172)$ & $\mathrm{CKD}(\mathrm{n}=385)$ & $P$-value \\
\hline Age (year) & $59.0[43.0,70.0]$ & $75.00[69.0,81.0]$ & $<0.001$ \\
\hline Sex, male & $40.1 \%$ & $49.9 \%$ & $<0.001$ \\
\hline BMI $\left(\mathrm{kg} / \mathrm{m}^{2}\right)$ & $22.9(3.7)$ & $23.8(3.9)$ & $<0.001$ \\
\hline SBP (mmHg) & $129.6(19.4)$ & $138.4(17.7)$ & $<0.001$ \\
\hline DBP (mmHg) & $78.7(11.5)$ & $78.8(11.3)$ & 0.841 \\
\hline \multicolumn{4}{|l|}{ Metabolic risk factor } \\
\hline Diabetes mellitus & $4.6 \%$ & $7.5 \%$ & 0.017 \\
\hline Dyslipidemia & $46.8 \%$ & $53.2 \%$ & 0.017 \\
\hline Hypertension & $54.0 \%$ & $61.8 \%$ & $<0.001$ \\
\hline Obesity & $25.6 \%$ & $33.8 \%$ & 0.001 \\
\hline \multicolumn{4}{|l|}{ Lifestyle } \\
\hline Regular alcohol intake & $38.0 \%$ & $29.9 \%$ & 0.002 \\
\hline Physical inactivity & $69.0 \%$ & $57.1 \%$ & $<0.001$ \\
\hline Insufficient sleep time & $5.8 \%$ & $7.3 \%$ & 0.252 \\
\hline \multicolumn{4}{|l|}{ Laboratory data } \\
\hline $\mathrm{Hb}(\mathrm{g} / \mathrm{dl})$ & $13.6(1.73)$ & $12.9(2.00)$ & $<0.001$ \\
\hline $\operatorname{Alb}(\mathrm{g} / \mathrm{dl})$ & $4.54(0.27)$ & $4.36(0.31)$ & $<0.001$ \\
\hline $\mathrm{Cr}(\mathrm{mg} / \mathrm{dl})$ & $0.66(0.13)$ & $1.12(0.77)$ & $<0.001$ \\
\hline $\mathrm{eGFR}\left(\mathrm{ml} / \mathrm{min} / 1.73 \mathrm{~m}^{2}\right)$ & $86.2(16.5)$ & $49.5(10.7)$ & $<0.001$ \\
\hline HDL-C (mg/dl) & $60.5(15.8)$ & $53.7(14.3)$ & $<0.001$ \\
\hline LDL-C (mg/dl) & $119.1(31.0)$ & $112.4(31.3)$ & $<0.001$ \\
\hline TG (mg/dl) & $110.0[75.0,169.0]$ & $128.0[89.0,175.0]$ & $<0.001$ \\
\hline HbA1c (NGSP, \%) & $5.2[4.9,5.4]$ & $5.4[5.1,5.7]$ & $<0.001$ \\
\hline \multicolumn{4}{|l|}{ Income(yen) } \\
\hline$<2,000,000$ & $12.3 \%$ & $18.4 \%$ & $<0.001$ \\
\hline$\geq 2,000,000$ & $87.7 \%$ & $81.6 \%$ & \\
\hline
\end{tabular}

Data are expressed as mean (standard deviation), mean\%, or median [25th and 75th percentiles].

the relationship between SES and lifestyle diseases, a gender difference in risk has been found [21]. But in our study, there was no significant interaction between sex and income $(P$-value $=0.402)$. Therefore, we have analyzed the adjusted ORs only in all subjects. Unadjusted analysis was used to calculate the odds ratios (ORs) and 95\% confidence intervals (95\% CIs) of CKD. Logistic regression analysis was used to calculate the ORs and 95\% CIs of CKD by income distinction after adjustment for age, hemoglobin (Hb), albumin (Alb), DM, DL, HT, obesity, regular alcohol intake and physical inactivity, with various models. As independent variables in the models, we selected those lifestyle and metabolic risks that were statistically significant (Table 1), as well as $\mathrm{Hb}$ and Alb, which 
are known risk factors for CKD.

Differences with a $P$-value $<0.05$ were considered statistically significant. All statistical analyses were performed using EZR (Version 1.33; Saitama Medical Center, Jichi Medical University, Saitama, Japan), which is a graphical user interface for R (The R Foundation for Statistical Computing, Vienna, Austria). More precisely, it is a modified version of $\mathrm{R}$ commander designed to add statistical functions frequently used in biostatistics [22].

Alb, albumin; BMI, body mass index; CKD, chronic kidney disease; $\mathrm{Cr}$, creatinine; DBP, diastolic blood pressure; eGFR, estimated glomerular filtration rate; $\mathrm{Hb}$, hemoglobin; HDL-C, high density lipoprotein cholesterol; LDL-C, low density lipoprotein cholesterol; NGSP, National Glycohemoglobin Standardization Program; SBP, systolic blood pressure; TG, triglyceride.

\section{Results}

The basic characteristics of the participants in terms of the presence of CKD are shown in Table 1. There were 3,172 participants without CKD, and 385 with CKD. The participants without CKD were younger and had lower SBP. Furthermore, those without CKD had higher levels of Hb, eGFR, Alb, HDL-C, and LDL-C, as well as lower levels of Cr, TG, and HbAlc. Regular alcohol intake, physical inactivity, and obesity were much more prevalent among patients without CKD. In addition, a higher proportion of individuals with CKD had an income of less than 2 million yen, and histories of DM, DL, and HT were also more prevalent.

The basic characteristics of the participants in terms of income are shown in Table 2. CKD, DM, DL, HT, and obesity were significantly more prevalent in the low income group.

Table 2. The basic characteristics of the participants in terms of income.

\begin{tabular}{cccc}
\hline Variables & $\begin{array}{c}\text { Income } \geq 2,000,000 \\
(\mathrm{n}=2538)\end{array}$ & $\begin{array}{c}\text { Income }<2,000,000 \\
(\mathrm{n}=1019)\end{array}$ & $P$-value \\
\hline Age (year) & $57.3(17.0)$ & $60.9(15.9)$ & $<0.001$ \\
Metabolic risk factor & & & \\
Chronic kidney disease & $9.9 \%$ & $13.1 \%$ & 0.09 \\
Diabetes mellitus & $4.7 \%$ & $6.4 \%$ & 0.032 \\
Dyslipidemia & $45.5 \%$ & $52.4 \%$ & $<0.001$ \\
Hypertension & $52.2 \%$ & $61.3 \%$ & $<0.001$ \\
Obesity & $25.5 \%$ & $29.0 \%$ & 0.032 \\
Lifestyle & & & $<0.001$ \\
Regular alcohol intake & $31.0 \%$ & $52.4 \%$ & 0.001 \\
Physical inactivity & $69.3 \%$ & $63.7 \%$ & 0.095 \\
Insufficient sleep time & $6.4 \%$ & $4.7 \%$ &
\end{tabular}

Data are expressed as mean \pm standard deviation or mean $\%$. 
Table 3 shows the unadjusted ORs and 95\% CIs of CKD. DM, DL, HT, and obesity were more prevalent in the CKD group. Regular alcohol intake and physical inactivity were significantly less common in the CKD group than in the non-CKD group. In the low income group, the prevalence of CKD was increased (OR: 1.61, 95\% CI: 1.20 - 2.14). There was no significant interaction between sex and income in our study $(P=0.4)$, although this result is not shown in the table. Thus, we only analyzed the adjusted OR using all subjects.

Table 4 shows the results of multivariate analysis adjusted using various models. The low income group was associated with an approximately $30 \%$ increase in the OR for CKD, after adjustment for age, sex, Hb, Alb, DM, DL, HT, and obesity (model 2 : $\mathrm{OR}=1.33,95 \% \mathrm{CI}=1.01-1.78$ ). When unhealthy lifestyle factors were added to the model, the association between income and the OR for CKD was not significant, but it was similar to model 2 (model 3: OR $=1.29,95 \% \mathrm{CI}=$ 0.96 - 1.73).

There were many data were missing in the present study. 3,557 people were

Table 3. Results of unadjusted odd ratio (OR) and $95 \%$ confidence interval (95\% CI) of CKD.

\begin{tabular}{lcc}
\hline \multicolumn{1}{c}{ Variables } & OR & $95 \% \mathrm{CI}$ \\
\hline Income (<2,000,000/ $\geq 2,000,000$ yen) & 1.61 & $1.20-2.14$ \\
Diabetes mellitus (yes/no) & 1.69 & $1.08-2.57$ \\
Dyslipidemia (yes/no) & 1.30 & $1.04-1.61$ \\
Hypertension (yes/no) & 1.34 & $1.10-1.72$ \\
Obesity (yes/no) & 1.48 & $1.17-1.86$ \\
Regular alcohol intake (yes/no) & 0.70 & $0.55-0.88$ \\
Physical inactivity (yes/no) & 0.60 & $0.48-0.75$ \\
Insufficient sleep time (yes/no) & 1.28 & $0.82-1.95$ \\
\hline
\end{tabular}

Table 4. Results of adjusted odd ratio (OR) and $95 \%$ confidence interval (95\% CI) of CKD.

\begin{tabular}{lccccccc}
\hline & \multicolumn{2}{c}{ Model 1 } & \multicolumn{2}{c}{ Model 2 } & \multicolumn{2}{c}{ Model 3 } \\
\cline { 2 - 7 } & OR & $95 \%$ CI & OR & $95 \%$ CI & OR & $95 \%$ CI \\
\hline Income (<2,000,000/ $\geq 2,000,000$ yen) & 1.60 & $1.21-2.11$ & 1.33 & $1.01-1.78$ & 1.29 & $0.96-1.72$ \\
Diabetes mellitus (yes/no) & 1.58 & $1.04-2.39$ & 1.27 & $1.01-1.59$ & 1.08 & $0.69-1.7$ \\
Dyslipidemia (yes/no) & 1.23 & $0.99-1.53$ & 1.15 & $0.74-1.82$ & 1.25 & $0.99-1.57$ \\
Hypertension (yes/no) & 1.30 & $1.04-2.39$ & 1.34 & $1.06-1.69$ & 1.32 & $1.04-1.67$ \\
Obesity (yes/no) & 1.50 & $1.17-1.92$ & 1.63 & $1.17-1.90$ & 1.52 & $1.19-1.94$ \\
\hline
\end{tabular}

Model 1 includes income, age, and sex; Model 2 includes model 1, hemoglobin level, albumin level, diabetes mellitus, dyslipidemia, hypertension, and obesity; Model 3 includes model 2, regular alcohol intake and physical inactivity. 
undergoing a blood test and 8,762 people did not. Though the result was not showed in table, we compared the age, sex, and income of people who had undergone a blood test with those of people who had eschewed a blood test. Those with blood results were older than those without $(51.3 \pm 23.2$ years vs. $44.3 \pm$ 23.6 years, $P<0.001)$. However, sex $(47.2 \%$ vs. $46.8 \%$ men, $P=0.7)$ and lower income status $(26.0 \%$ vs. $23.5 \%, P=0.1)$ were similar between the two groups.

\section{Discussion}

In Western countries, SES is associated with CKD prevalence, progression, and risk of mortality. However, in Japan, the association is not clear. We investigated the association between individual income status-a major indicator of SES-and CKD in Japan. The results of the present study indicate that people of lower income have an approximately $30 \%$ greater prevalence of CKD than those of higher income in multivariate regression analysis, after adjustment for age, sex, Hb, Alb, DM, DL, HT, and obesity.

In American and British studies, CKD has been strongly related to both lifestyle factors and SES indicators, such as income, education, and occupation. For instance, Merkin et al. indicated that residency in an area of low SES is independently associated with a greater risk of progressive CKD [14]. Similarly, in a 3 -year follow-up study, Krop et al. suggested that kidney function decline is 2.83 times more likely to develop in those who have a household income of less than $\$ 16,000$ per year than in those whose household income is $\$ 35,000$ per year or more [16]. The present study corroborated these previous investigations in finding a relationship between lower income and CKD. To our knowledge, the present study was the first to examine the relationship between individual income and CKD in Japan, and the results were similar to those of previous studies in other countries. That is, the odds of CKD in the $<2$ million yen group (almost 17,000 US dollars) were 1.3 times higher than those in the $\geq 2$ million yen group.

There are several reasons why SES may influence the prevalence of CKD. Firstly, unhealthy lifestyles and metabolic factors may contribute to the association between SES and CKD. In previous reports, many people with low SES have an unhealthy lifestyle [21] [23] [24]. Priya et al. showed that proportion of participants with unhealthy lifestyles, such as high alcohol intake and physical inactivity were higher in participants with low SES [25]. It may be that metabolic risks, such as DM, DL, HT and obesity, in addition to unhealthy lifestyle factors, are mediators of the association between low SES and CKD [25]. In summary, unhealthy lifestyles and metabolic risk factors may develop due to low SES, resulting in CKD. In the present study, similar results were found. Those with low income had tended to have unhealthy lifestyles and metabolic risk factors. And the results of the present study indicate that people with metabolic risk such DM, DL, HT, and obesity, have greater prevalence of CKD than those who did not have. There was an association between low income and CKD in multivariate analysis, after adjustment for unhealthy lifestyle and metabolic risks. 
Therefore, to prevent CKD, it is necessary to improve lifestyle-related diseases and unhealthy habits, especially among those with lower income. Secondly, it may be that uncollected factors affect the onset of CKD, such as stress caused by low income, or lack of access to medical services. In this regard, it was interesting that we found a relationship between income and CKD, even in Japan, where healthcare is easy to access. In a past study, health insurance and routine healthcare services were examined as potential meditators of the SES-CKD association, as were health-related behaviors and comorbid conditions [25]. Factors related to healthcare access contributed $11 \%$ to the SES-CKD association, indicating that the contribution of healthcare factors is greater than that of health-related behaviors such as smoking or alcohol intake. Conversely, Japan, which has universal healthcare coverage (UHC), has significant equity in its healthcare system, expanding coverage to almost all citizens [26]. One major strength of our study was that it showed that, even in country with UHC, lower income may be a risk for CKD.

Accordingly, advancing our understanding of the role of socioeconomic factors in the onset and progression of CKD should lead to improved strategies for disease prevention and management. Specifically, clinicians could prevent CKD by modifying the health services that are available through UHC, considering that patients with CKD are often in low income strata in Japan. In this regard, although there is UHC in Japan, it may be difficult to access because few health services are covered and many people with low income still have to finance their own treatment. This is now considered a serious problem, because a lack of access to medical services may be a mechanism of CKD development. To break this barrier to access, it may be effective to remunerate health check-ups; that is, if people were paid to seek medical check-ups, or if the self-burden of medical expenses were reduced, people with low SES may more readily seek health check-ups or visit routine medical care. CKD is associated with SES, even after adjustment for those metabolic risks that were assessed at medical examination. However, the rate of specific health check-ups and special health-maintenance guidance is high in large companies, while the rate is low in small and medium enterprises, as well as among the self-employed. In addition, it is difficult for people of low SES to access medical services [27]. Secondly, a population-approach is important in the prevention CKD. As we have stated above, the prevalence of CKD is, for many reasons, higher among individuals of lower SES. In turn, a population-approach will reduce risk factors and thus increase the health level of the entire population; that is, it may be possible to prevent the onset of CKD, even among people of low SES. Case in point, in co-operation with a private company, the United Kingdom reduced the national salt intake by reducing the amount of salt in processed foods [28]. Salt reduction activity for all citizens-a population-approach-may prevent unhealthy lifestyle, which is one of CKD risk factors, in the future. We should formulate and implement more such strategies to stem the tide of CKD. 
There were several limitations to the present study. Firstly, it was a cross-sectional study, and causal inferences could not be made because we could not determine temporal sequence. Secondly, we used income as the sole indicator of SES, because other indicators, such as education and occupation, were not collected in the survey. In general, education and occupational history should also be considered. Future studies should examine the relationship between occupation or education and CKD. Thirdly, there may have been selection bias, because many data were missing in the present study. For instance, the patients needed to visit a designated laboratory for blood tests. However, not many people came to this laboratory, and thus the amount of missing data was increased. As a result of comparing the attributes of the person undergoing blood test, missing data were not considered to have a significant effect on the results of the present study. Fourthly, our data did not include a few critical factors for cofounding and medicating such as smoking status, unhealthy diet and stress. These unhealthy factors may contribute to the association between SES and CKD. According to Fukuda et al., the smoking rate in men is 2.03 times higher among lower income classes than among higher classes [21]. Keita et al. showed that children in disadvantaged neighborhoods consumed a greater percentage of calories in the form of fats and trans fats, and that they had a higher sodium intake [23] [24]. The study including these factors will elucidate the pathway from SES to CKD more clearly. Lastly, because CKD is described as an aging-related phenomenon rather than a disease, it may be over-diagnosed in elderly people, who are also likely to have lower income than younger participants. Indeed, some studies have cast doubt on whether the GFR can be estimated using serum $\mathrm{Cr}$ in elderly patients with CKD [29]. That said, to estimate the GFR among elderly Japanese patients, we used a formula that the Japanese Society of Nephrology has judged as valid. It is important that future researchers continue working to assess the relationship between income and GFR, which can be estimated more accurately using serum cystatin [30].

\section{Conclusion}

In conclusion, the present study revealed that low income is significantly related to CKD in Japan, which there is UHC. Further studies are needed to assess the association between temporal variations in SES and CKD prevalence. Additionally, interventions such as a population-approach should be considered.

\section{Acknowledgements}

We thank the faculty and students of the Graduate School of Public Health, Teikyo University, for their comments and discussions.

\section{Competing Interests}

The authors declare that they have no conflicts of interest in relation to this article. 


\section{Funding}

This study was supported by a Grant-in-Aid for Scientific Research (C) (No. 25460755 and No. 16K09073) from the Japan Society for the Promotion of Science, Japan, and by a Health and Labor Sciences Research Grant (H25-Jyunkanki-Ippan-006]) from the Ministry of Health, Labor and Welfare, Japan.

\section{References}

[1] Liyanage, T., Ninomiya, T., Jha, V., Neal, B., Patrice, H.M., Okpechi, I., et al. (2015) Worldwide Access to Treatment for End-stage Kidney Disease: A Systematic Review. Lancet, 385, 1975-1982. https://doi.org/10.1016/S0140-6736(14)61601-9

[2] CKD Guideline Editorial Committee (2009) Evidence-Based Practice Guideline for the Treatment of CKD. Nihon Jinzo Gakkai Shi, 51, 905-1066 (in Japanese).

[3] Japanese Society for Dialysis Therapy (JSDT) (2015) The Interpretation and Reporting of These Data Are the Responsibility of the Authors and in No Way Should Be Seen as an Official Policy or Interpretation of the JSDT. http://docs.jsdt.or.jp/overview/index.html

[4] Ritz, E. (2003) Minor Renal Dysfunction: An Emerging Independent Cardiovascular Risk Factor. Heart, 89, 963-964. https://doi.org/10.1136/heart.89.9.963

[5] Ruilope, L.M., van Veldhuisen, D.J., Ritz, E. and Luscher, T.F. (2001) Renal Function: The Cinderella of Cardiovascular Risk Profile. Journal of the American College of Cardiology, 38, 1782-1787.

[6] Shoji, T., Abe, T., Matsuo, H., Egusa, G., Yamasaki, Y., Kashihara, N., et al. (2012) Chronic Kidney Disease, Dyslipidemia, and Atherosclerosis. Journal of Atherosclerosis and Thrombosis, 19, 299-315. https://doi.org/10.5551/jat.10454

[7] Ohkubo, Y., Kishikawa, H., Araki, E., Miyata, T., Isami, S., Motoyoshi, S., et al. (1995) Intensive Insulin Therapy Prevents the Progression of Diabetic Microvascular Complications in Japanese Patients with Non-Insulin-Dependent Diabetes Mellitus: A Randomized Prospective 6-Year Study. Diabetes Research and Clinical Practice, 28, 103-117. https://doi.org/10.1016/0168-8227(95)01064-K

[8] Schaeffner, E.S., Kurth, T., Curhan, G.C., Glynn, R.J., Rexrode, K.M., Baigent, C., et al. (2003) Cholesterol and the Risk of Renal Dysfunction in Apparently Healthy Men. Journal of the American Society of Nephrology, 14, 2084-2091.

[9] Bakris, G.L., Williams, M., Dworkin, L., Elliott, W.J., Epstein, M., Toto, R., et al. (2000) Preserving Renal Function in Adults with Hypertension and Diabetes: A Consensus Approach. American Journal of Kidney Diseases, 36, 646-661. https://doi.org/10.1053/ajkd.2000.16225

[10] Stengel, B., Tarver-Carr, M.E., Powe, N.R., Eberhardt, M.S., and Brancati, F.L. (2003) Lifestyle Factors, Obesity and the Risk of Chronic Kidney Disease. Epidemiology, 14, 479-487. https://doi.org/10.1097/01.EDE.0000071413.55296.c4

[11] Yamamoto, R., Nagasawa, Y., Iwatani, H., Shinzawa, M., Obi, Y., Teranishi, J., et al. (2012) Self-Reported Sleep Duration and Prediction of Proteinuria: A Retrospective Cohort Study. American Journal of Kidney Diseases, 59, 343-355. https://doi.org/10.1053/j.ajkd.2011.08.032

[12] Perneger, T.V., Whelton, P.K., Puddey, I.B. and Klag, M.J. (1999) Risk of End-Stage Renal Disease Associated with Alcohol Consumption. American Journal of Epidemiology, 150, 1275-1281. https://doi.org/10.1093/oxfordjournals.aje.a009958 
[13] Wakasugi, M., Kazama, J.J., Yamamoto, S., Kawamura, K. and Narita, I. (2013) A Combination of Healthy Lifestyle Factors Is Associated with a Decreased Incidence of Chronic Kidney Disease: A Population-Based Cohort Study. Hypertension Research, 36, 328-333. https://doi.org/10.1038/hr.2012.186

[14] Merkin, S.S., Coresh, J., Diez Roux, A.V., Taylor, H.A. and Powe, N.R. (2005) Area Socioeconomic Status and Progressive CKD: The Atherosclerosis Risk in Communities (ARIC) Study. American Journal of Kidney Diseases, 46, 203-213. https://doi.org/10.1053/j.ajkd.2005.04.033

[15] Krop, J.S., Coresh, J., Chambless, L.E., Shahar, E., Watson, R.L., Szklo, M., et al. (1999) A Community-Based Study of Explanatory Factors for the Excess Risk for Early Renal Function Decline in Blacks vs Whites with Diabetes: The Atherosclerosis Risk in Communities Study. Archives of Internal Medicine, 159, 1777-1783. https://doi.org/10.1001/archinte.159.15.1777

[16] Merkin, S.S., Diez Roux, A.V., Coresh, J., Fried, L.F., Jackson, S.A. and Powe, N.R. (2007) Individual and Neighborhood Socioeconomic Status and Progressive Chronic Kidney Disease in an Elderly Population: The Cardiovascular Health Study. Social Science \& Medicine, 65, 809-821. https://doi.org/10.1016/j.socscimed.2007.04.011

[17] Ministry of Health Labour and Welfare (2011) 2010 Comprehensive Survey of the Living Conditions of People on Health and Welfare. Health and Welfare Statistics Association, Tokyo.

[18] Tajima, N., Noda, M., Origasa, H., Noto, H., Yabe, D., Fujita, Y., et al. (2015) Evidence-Based Practice Guideline for the Treatment for Diabetes in Japan 2013. Diabetology International, 6, 151-187. https://doi.org/10.1007/s13340-015-0206-2

[19] Japan Atherosclerosis Society (2007) Japan Atherosclerosis Society (JAS) Guidelines for Prevention of Atherosclerotic Cardiovascular Diseases. Journal of Atherosclerosis and Thrombosis, 5-57. (In Japanese)

[20] Shimamoto, K., Ando, K., Fujita, T., Hasebe, N., Higaki, J., Horiuchi, M., et al. (2014) The Japanese Society of Hypertension Guidelines for the Management of Hypertension (JSH 2014). Hypertension Research, 37, 253-390. https://doi.org/10.1038/hr.2014.20

[21] Fukuda, Y., Nakamura, K. and Takano, T (2005) Accumulation of Health Risk Behaviours Is Associated with Lower Socioeconomic Status and Women's Urban Residence: A Multilevel Analysis in Japan. BMC Public Health, 5, 53.

https://doi.org/10.1186/1471-2458-5-53

[22] Kanda, Y. (2013) Investigation of the Freely Available Easy-to-Use Software 'EZR' for Medical Statistics. Bone Marrow Transplantation, 48, 452-458. https://doi.org/10.1038/bmt.2012.244

[23] Keita, A.D., Casazza, K., Thomas, O. and Fernandez, J.R. (2009) Neighborhood-Level Disadvantage Is Associated with Reduced Dietary Quality in Children. Journal of the American Diabetic Association, 109, 1612-1616. https://doi.org/10.1016/j.jada.2009.06.373

[24] Gray, L. and Leyland, A.H. (2009) A Multilevel Analysis of Diet and Socio-Economic Status in Scotland: Investigating the 'Glasgow Effect'. Public Health Nutrition, 12, 1351-1358. https://doi.org/10.1017/S1368980008004047

[25] Vart, P., Gansevoort, R.T., Crews, D.C., Reijneveld, S.A. and Bültmann, U. (2015) Mediators of the Association between Low Socioeconomic Status and Chronic Kidney Disease in the United States. American Journal of Epidemiology, 181, 385-396. https://doi.org/10.1093/aje/kwu316 
[26] Ikegami, N., Yoo, B.K., Hashimoto, H., Matsumoto, M., Ogata, H., Babazono, A., et al. (2011) Japanese Universal Health Coverage: Evolution, Achievements, and Challenges. Lancet, 378, 1106-1115. https://doi.org/10.1016/S0140-6736(11)60828-3

[27] Baker, D. and Middleton, E. (2003) Cervical Screening and Health Inequality in England in the 1990s. Journal of Epidemiology and Community Health, 57, 417-423. https://doi.org/10.1136/jech.57.6.417

[28] Brinsden, H.C., He, F.J., Jenner, K.H. and Macgregor, G.A. (2013) Surveys of the S alt Content in UK Bread: Progress Made and Further Reductions Possible. BMJ Open, 3, pii: e002936. https://doi.org/10.1136/bmjopen-2013-002936

[29] Beddhu, S., Samore, M.H., Roberts, M.S., Stoddard, G.J., Pappas, L.M. and Cheung, A.K. (2003) Creatinine Production, Nutrition, and Glomerular Filtration Rate Estimation. Journal of the American Journal of Nephrology, 14, 1000-1005.

[30] Hoek, F.J., Kemperman, F.A. and Krediet, R.T. (2003) A Comparison between Cystatin C, Plasma Creatinine and the Cockcroft and Gault Formula for the Estimation of Glomerular Filtration Rate. Nephrology, Dialysis, Transplantation, 18, 2024-2031. https://doi.org/10.1093/ndt/gfg349 